\title{
Towards an Experimentally Simple Procedure for the Determination of Energy Transfer Parameters in Gain Media
}

\author{
Stephen J. Beecher, Jakub W. Szela and Jacob I. Mackenzie \\ Optoelectronics Research Centre, University of Southampton, Southampton, SO17 1BJ, UK \\ S.J.Beecher@soton.ac.uk
}

\begin{abstract}
A promising technique is presented for characterization of rates of energy transfer within solid-state laser crystals. The technique is applied to a 6 at. $\% \mathrm{Tm}^{3+}$ :YLF crystal pumped with a $1680 \mathrm{~nm}$ laser diode.

OCIS codes: (300.6280) Spectroscopy, fluorescence and luminescence; (300.6420) Spectroscopy, nonlinear.
\end{abstract}

\section{Introduction}

Accurate spectroscopic measurements of gain media are of key importance for assessing the suitability of materials for an intended application. Apart from the typical spectroscopic parameters needed to assess the potential quality of the materials gain, possible parasitic effects such as energy transfer upconversion (ETU) also need to be evaluated with sufficient accuracy to determine the impact it may have. An ideal measurement is quick to carry out and leads to the determination of the parameter in question with low uncertainty. A range of methods are routinely used for the determination of absorption and emission cross sections, and fluorescence lifetimes, allowing simple media to be accurately characterized. At higher concentrations a range of energy transfer mechanisms can occur, complicating any analysis. This is of particular significance for thulium $\left(\mathrm{Tm}^{3+}\right)$ systems where high dopant concentrations are often utilized to access efficient cross relaxation (CR) and effective pumping with well-developed $~ 800 \mathrm{~nm}$ highpower laser diodes. The tradeoff for efficient ${ }^{3} \mathrm{H}_{6},{ }^{3} \mathrm{H}_{4}>{ }^{3} \mathrm{~F}_{4},{ }^{3} \mathrm{~F}_{4}$ cross relaxation is an increased rate of the inverse process, ETU. Several methods have been proposed for measuring ETU rates, these typically require costly equipment producing short energetic pulses (typically $10 \mathrm{~ns}$, few $\mathrm{mJ}$ level) [1], or indirect measurement based on laser performance [2]. Here we employ a low cost, simple experimental method utilizing a laser diode to characterize the gain media and cross relaxation and ETU rates. This technique has been applied to a 6at.\% $\mathrm{Tm}^{3+}: \mathrm{YLF}$ crystal by exciting population into the first excited state manifold, ${ }^{3} \mathrm{~F}_{4}$, with a QCW $1680 \mathrm{~nm}$ laser diode and observing fluorescence from both the ${ }^{3} \mathrm{~F}_{4}$ manifold at approximately $2000 \mathrm{~nm}$ and the third excited state manifold, ${ }^{3} \mathrm{H}_{4}$ with emission close to $800 \mathrm{~nm}$.

\section{Rate equations}

For this work we have assumed that only the four lowest manifolds of the thulium ${ }^{3+}$ ion are populated; all higher levels are neglected. The populations of the different energy levels within the system are assumed to be accurately described by the set of rate equations presented below. For brevity the populations of the ${ }^{3} \mathrm{H}_{6},{ }^{3} \mathrm{~F}_{4},{ }^{3} \mathrm{H}_{5}$ and ${ }^{3} \mathrm{H}_{4}$ manifolds are labeled $\mathrm{n}_{1}, \mathrm{n}_{2}, \mathrm{n}_{3}$ and $\mathrm{n}_{4}$ respectively and population in the ${ }^{3} \mathrm{H}_{5}$ manifold is assumed to quickly relax into the ${ }^{3} \mathrm{~F}_{4}$ manifold:

$$
\begin{aligned}
& \frac{d n_{1}}{d t}=\mathrm{A}_{21} \cdot \mathrm{n}_{2}+\mathrm{A}_{41} \cdot \mathrm{n}_{4}-\sigma_{12} \cdot \mathrm{I}_{\mathrm{p}} \cdot \mathrm{n}_{1}+\sigma_{21} \cdot \mathrm{I}_{\mathrm{p}} \cdot \mathrm{n}_{2}+\mathrm{w}_{\text {etu }} \cdot \mathrm{n}_{2}{ }^{2}-\mathrm{w}_{\text {cross }} \cdot \mathrm{n}_{1} \cdot \mathrm{n}_{4} \\
& \frac{d n_{2}}{d t}=-\mathrm{A}_{21} \cdot \mathrm{n}_{2}+\left(\mathrm{A}_{42}+\mathrm{A}_{43}\right) \cdot \mathrm{n}_{4}+\sigma_{12} \cdot \mathrm{I}_{\mathrm{p}} \cdot \mathrm{n}_{1}-\sigma_{21} \cdot \mathrm{I}_{\mathrm{p}} \cdot \mathrm{n}_{2}-2 \cdot \mathrm{w}_{\mathrm{etu}} \cdot \mathrm{n}_{2}{ }^{2}+2 \cdot \mathrm{w}_{\text {cross }} \cdot \mathrm{n}_{1} \cdot \mathrm{n}_{4} \\
& \frac{d n_{4}}{d t}=-\mathrm{A}_{41} \cdot \mathrm{n}_{4}-\left(\mathrm{A}_{42}+\mathrm{A}_{43}\right) \cdot \mathrm{n}_{4}+\mathrm{w}_{\text {etu }} \cdot \mathrm{n}_{2}{ }^{2}-\mathrm{w}_{\text {cross }} \cdot \mathrm{n}_{1} \cdot \mathrm{n}_{4}
\end{aligned}
$$

Where $A_{i j}$ describes the rate of decay of population from energy level $i$ to energy level $j, \sigma_{12}$ denotes the effective absorption cross section of the ground state at the laser diode wavelength and $\sigma_{21}$ denotes the effective emission cross section of the ${ }^{3} \mathrm{~F}_{4}$ level at the laser diode wavelength. $\mathrm{I}_{\mathrm{p}}$ denotes the effective pump-photon flux, the number of photons passing through an area of $1 \mathrm{~cm}^{2}$ each second multiplied by the material's refractive index. The $\mathrm{W}_{\text {etu }}$ and $\mathrm{W}_{\text {cross }}$ parameters account for ETU and CR. 
For boundary conditions it is assumed that at $\mathrm{t}=0$, a long time after any previous optical excitation all the ions are in the ground state, ie. $\mathrm{n}_{1}=\mathrm{n}_{\text {total }}$ and $\mathrm{n}_{2}=\mathrm{n}_{3}=\mathrm{n}_{4}=0$. An energy level diagram with the modeled transitions is presented in Fig 1(a). For accuracy between simulated and experimental results it is necessary to accurately establish each of the parameters in the rate equations. Literature values of radiative lifetimes for Tm:YLF are complicated by significant overlap between emission and absorption spectra leading to strong radiation trapping. For our lifetime measurements the 'pinhole method' was employed to mitigate against this energy migration mechanism [3]. This technique allows us to measure $\mathrm{a}^{3} \mathrm{~F}_{4}$ lifetime of $6.9 \pm 0.1 \mathrm{~ms}$, significantly shorter than the $12.5 \mathrm{~ms}$ we measure with no pinhole. For the branching ratios used to calculate the rates we use the values given by Walsh et al [4].

a

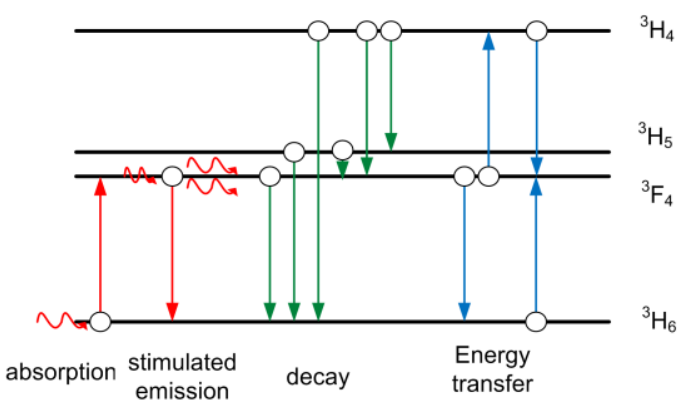

b

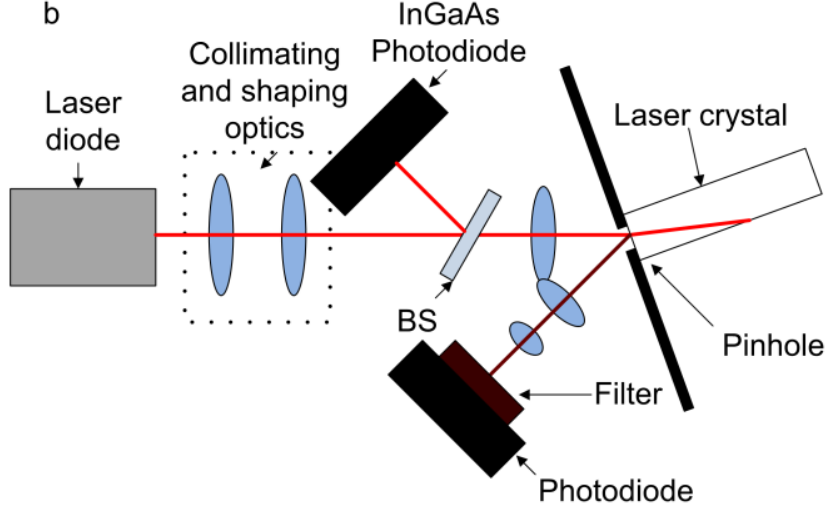

Fig 1. (a) Energy level diagram for $\mathrm{Tm}^{3+}$ featuring the modeled transitions including energy transfer upconversion ( $2^{\text {nd }}$ from right) and cross relaxation (far right). (b) The experimental setup: BS is a beam splitter, directing a fraction of the pump beam onto a photodiode. The other photodiode is appropriately filtered to only measure the desired radiative transition.

\section{Experimental setup}

A 1680nm single emitter laser diode is collimated by two cylindrical lenses and a small fraction of the light is reflected onto an InGaAs photodiode to allow the pump signal to be recorded. The remainder of the light is focused to a $\sim 150 \mu \mathrm{m}$ radius spot close to the surface of the crystal under test. The crystal is placed at an angle of $\sim 35^{\circ}$ to normal incidence and a pinhole is placed in contact with the crystal face. While the pinhole may act to limit radiation trapping its main role is to block fluorescence signal from regions of crystal experiencing lower intensities due to prior absorption and divergence of the pump beam. The $1.5 \mathrm{~mm}$ diameter pinhole used is estimated to reduce the observation depth to around $1.3 \mathrm{~mm}$, comparable to the small signal absorption length of $1.1 \mathrm{~mm}$. A single plano convex lens collects fluorescence, which is focused through an appropriate filter onto a photodiode. The 800nm fluorescence is collected with a silicon photodiode and the $2000 \mathrm{~nm}$ fluorescence with an extended InGaAs photodiode. The photodiode signals are amplified and recorded using a digital oscilloscope. The pump output is controlled by a laser diode driver, modulated by a pulse generator capable of varying the duration of the laser pulses. A drive current was found such that the pulse duration could be varied between $3 \mathrm{~ms}$ and $50 \mathrm{~ms}$, with minimal change in the laser spectrum at a repetition frequency of $3 \mathrm{~Hz}$. The signals from the photodiodes were recorded for pump durations of 3, 5, 10, 25 and 50ms and are shown, normalized to the highest value, in Fig 2.
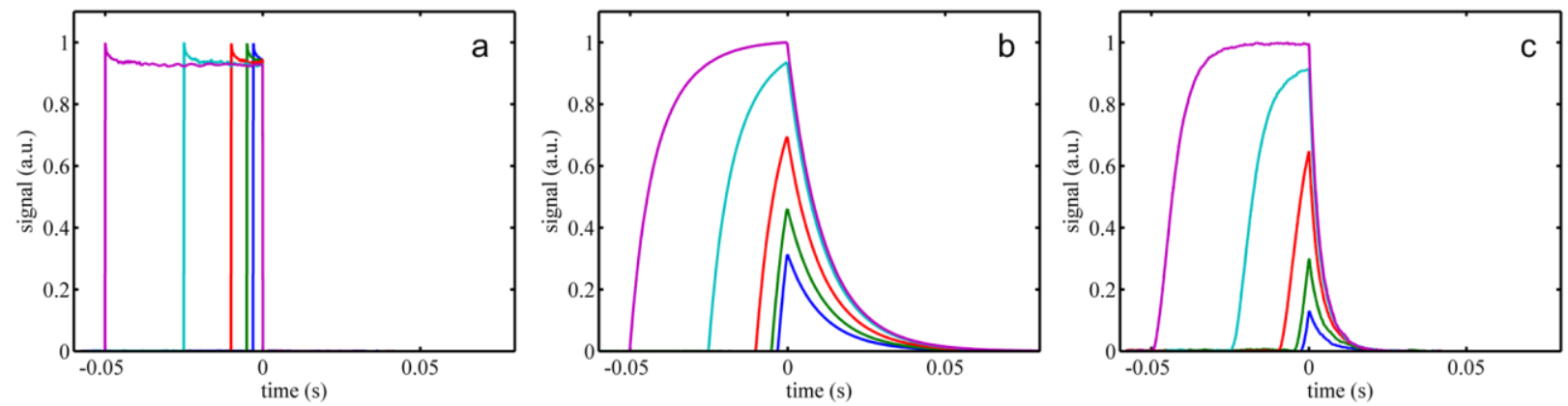

Fig 2. (a) The signal from the photodiode measuring the temporal pulse profile of the pump. (b) The temporal profile of the 2000nm fluorescence. (c) The temporal profile of the $800 \mathrm{~nm}$ fluorescence. All are plotted on linear scales.

\section{Analysis}


The rate equations described above were numerically solved and compared to experimental data for a range of $\mathrm{W}_{\text {cross }}, \mathrm{W}_{\text {etu }}$ and $\mathrm{I}_{\mathrm{p}}$ parameters. The $\mathrm{CR}$ parameter strongly shapes the decay of the ${ }^{3} \mathrm{H}_{4}$ population, no accurate fit could be found for values less than $10^{-18} \mathrm{~cm}^{3} \cdot \mathrm{s}^{-1}$ imposing a hard lower limit on this value. The ETU parameter shapes the relative peak fluorescence values for the different excitation durations and no fit could be found for values higher than $10^{-18} \mathrm{~cm}^{3} \cdot \mathrm{s}^{-1}$ imposing an upper limit on this energy transfer mechanism. The effect on the simulated ${ }^{3} \mathrm{H}_{4}$ population of changing the ETU and CR parameters over 3 orders of magnitude is shown in figure 3.
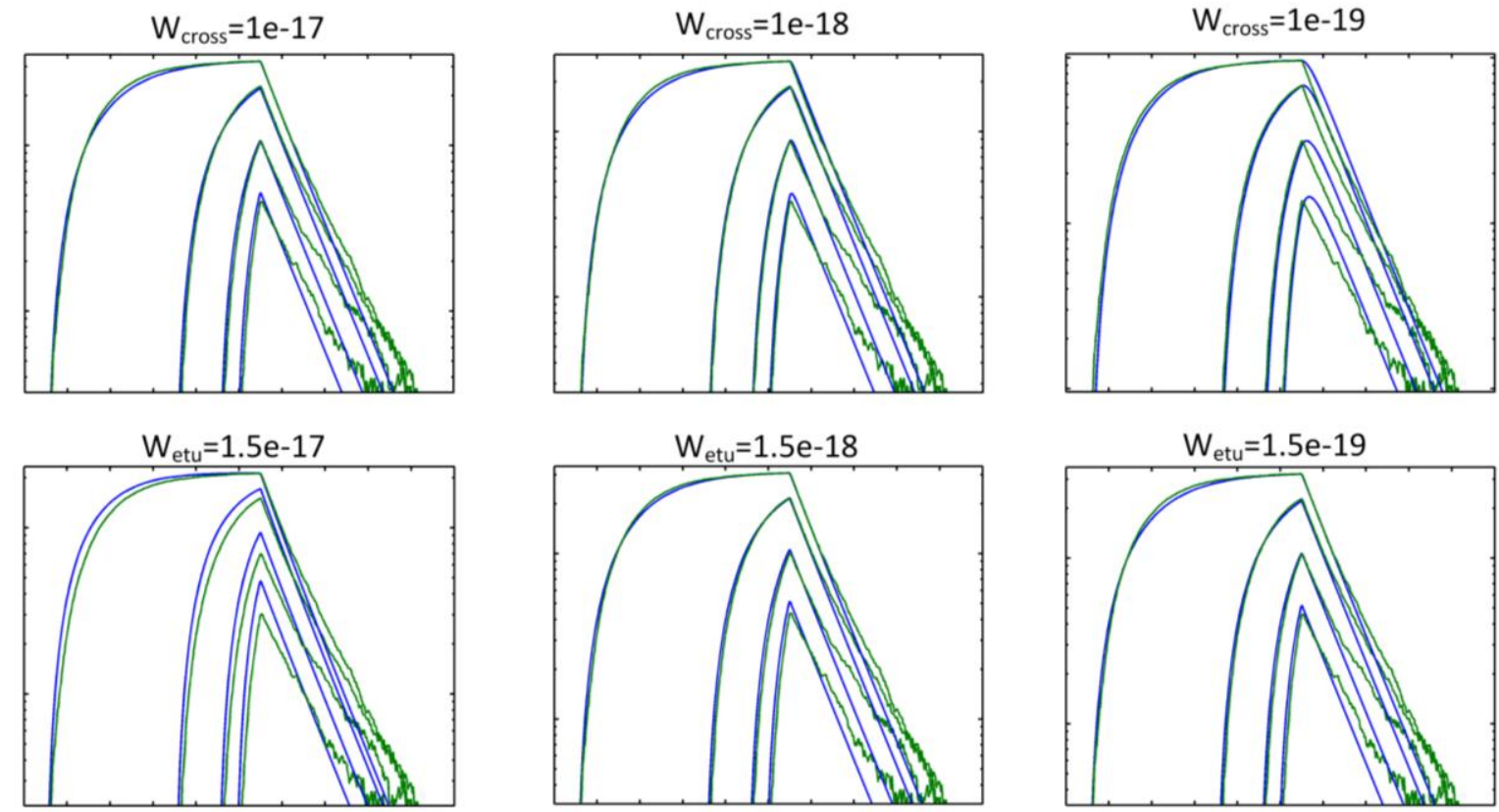

Fig 3. The effect of $\mathrm{CR}$ and $\mathrm{ETU}$ on the $3 \mathrm{H} 4$ fluorescence. Top $\mathrm{W}_{\text {cross }}$ variation, green is experimental data, blue is simulation. Bottom $\mathrm{W}_{\text {etu }}$ variation, green is experimental data, blue is simulation. All are plotted with logarithmic y scale for an irradiance of $135 \mathrm{~W} / \mathrm{cm}^{2}$.

Several assumptions made within the model have not yet been fully realized experimentally. One of potential significance is the spatial dependence of the pump irradiance. In this work the pump beam was focused to a spot size significantly smaller than the pinhole used, with an average irradiance of $2 \mathrm{~kW} \cdot \mathrm{cm}^{-2}$, with a near-Gaussian pump profile. Due to the nonlinear nature of the dynamics of the system this effect will manifest differently in the fluorescence waveforms from the ${ }^{3} \mathrm{H}_{4}$, which is populated non-linearly, and ${ }^{3} \mathrm{~F}_{4}$ manifold, where the predominant population and depopulation mechanisms are linear. Several techniques are under investigation to reduce the effect of the non-uniform pump irradiance.

\section{Summary}

We have employed a simple technique to determine an upper bound for the ETU and lower bound on the CR rates for $6 \% \mathrm{Tm}$ :YLF. With refinement of the technique a significant reduction in the uncertainty is expected. The experimental equipment required is readily available in most optics labs and the time required for measurements is minimal potentially making this an attractive technique for the rapid characterization of gain media.

\section{References}

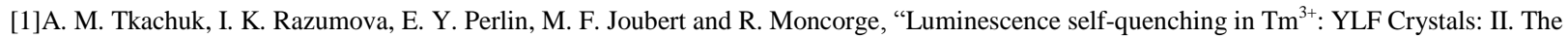
luminescence decay and macrorates of energy transfer," Opt Spectrosc. 90, 1 (2001).

[2] J. W. Kim, J. I. Mackenzie and W. A. Clarkson, "Influence of energy-transfer-upconversion on threshold pump power in quasi-three-level solid-state lasers," Opt. Express 17, 14, 11935-11943 (2009).

[3] H. Kühn, S. T. Fredrich-Thornton, C. Kränkel, R. Peters and K. Petermann, "Model for the calculation of radiation trapping and description of the pinhole method," Opt. Lett. 32, 13 (2007).

[4] B. M. Walsh, N. P. Barnes and B. D. Bartolo, "Branching ratios, cross sections, and radiative lifetimes of rare earth ions in solids: Application to $\mathrm{Tm}^{3+}$ and $\mathrm{Ho}^{3+}$ ions in $\mathrm{LiYF}_{4}$," J. Appl. Phys. 83, 2772 (1998). 